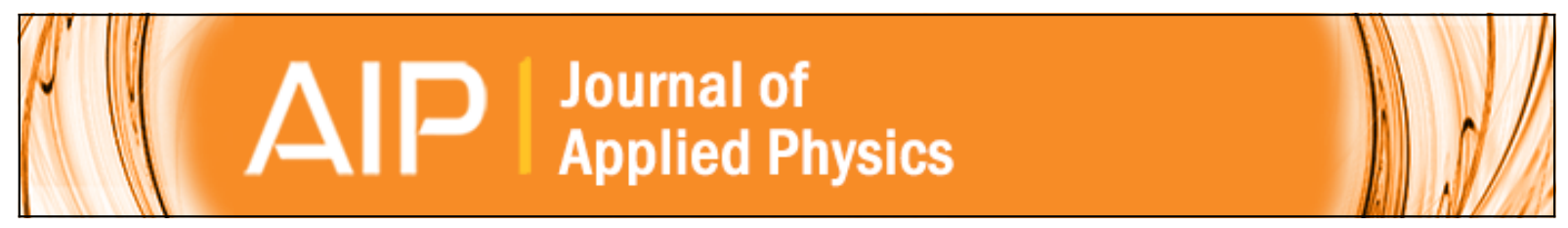

\title{
Analysis of terahertz generation via nanostructure enhanced plasmonic excitations
}

Yaohui Gao, Meng-Ku Chen, Chia-En Yang, Yun-Ching Chang, Stuart Yin, Rongqing Hui, Paul Ruffin, Christina

Brantley, Eugene Edwards, and Claire Luo

Citation: Journal of Applied Physics 106, 074302 (2009); doi: 10.1063/1.3236629

View online: http://dx.doi.org/10.1063/1.3236629

View Table of Contents: http://scitation.aip.org/content/aip/journal/jap/106/7?ver=pdfcov

Published by the AIP Publishing

\section{Articles you may be interested in}

Spontaneous emission and collection efficiency enhancement of single emitters in diamond via plasmonic cavities and gratings

Appl. Phys. Lett. 103, 161101 (2013); 10.1063/1.4817397

Polymeric photovoltaics with various metallic plasmonic nanostructures

J. Appl. Phys. 113, 063109 (2013); 10.1063/1.4790504

Spectral analysis of enhanced third harmonic generation from plasmonic excitations

Appl. Phys. Lett. 98, 261909 (2011); 10.1063/1.3604794

Silver half-shell arrays with controlled plasmonic response for fluorescence enhancement optimization Appl. Phys. Lett. 95, 193110 (2009); 10.1063/1.3263193

Excitation of dielectric-loaded surface plasmon polariton observed by using near-field optical microscopy Appl. Phys. Lett. 93, 073306 (2008); 10.1063/1.2973355

\section{AIP $\left.\right|_{\text {APL Photonics }}$}

APL Photonics is pleased to announce Benjamin Eggleton as its Editor-in-Chief

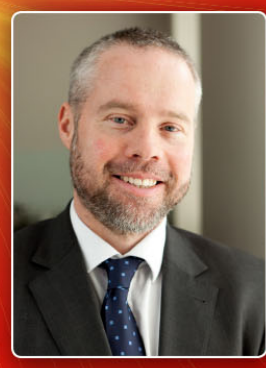




\title{
Analysis of terahertz generation via nanostructure enhanced plasmonic excitations
}

\author{
Yaohui Gao, ${ }^{1}$ Meng-Ku Chen, ${ }^{1}$ Chia-En Yang, ${ }^{1}$ Yun-Ching Chang, ${ }^{1}$ Stuart Yin, ${ }^{1, a)}$ \\ Rongqing Hui, ${ }^{2}$ Paul Ruffin, ${ }^{3}$ Christina Brantley, ${ }^{3}$ Eugene Edwards, ${ }^{3}$ and Claire Luo ${ }^{4}$ \\ ${ }_{1}^{1}$ Department of Electrical Engineering, Penn State University, University Park, Pennsylvania 16802, USA \\ ${ }^{2}$ Department of Electrical Engineering and Computer Science, The University of Kansas, Lawrence, \\ Kansas 66045, USA \\ ${ }^{3}$ US Army Aviation and Missile Research Development and Engineering Center, Redstone Arsenal, Alabama \\ 35898, USA \\ ${ }^{4}$ General Opto Solutions, LLC State College, Pennsylvania 16803, USA
}

(Received 21 June 2009; accepted 26 August 2009; published online 1 October 2009)

\begin{abstract}
In this paper, we conduct a quantitative study on the physical mechanism of electrons dynamics near the nanostructured metal film surfaces, as well as the efficiency of generated terahertz radiation associated with different types of nanostructures. The simulation results show that although the oscillating motion of emitted electrons outside the metal surface may affect the terahertz generation efficiency to some extent, this efficiency is predominantly determined by the electric field magnitude inside the metal film associated with nanostructure enhanced plasmonic excitations. Due to the field enhancement effect of the nanostructure, an appropriately designed nanostructured surface could greatly enhance the strength of generated terahertz signal via the increased nonlinear interactions between the light and the nanostructures. (C) 2009 American Institute of Physics. [doi:10.1063/1.3236629]
\end{abstract}

\section{INTRODUCTION}

Terahertz wave radiation from nanostructured metallic surfaces was observed recently. ${ }^{1}$ This phenomenon could be explained by the electron accelerations due to the ponderomotive potential associated with surface plasmon excitation. There are numerous applications in this spectral range, such as terahertz imaging, ${ }^{2}$ spectroscopy, ${ }^{3}$ and material characterization. ${ }^{4}$ Terahertz emission can be produced based on different physical mechanisms. One such example is terahertz generation involving photoconductive antenna that relies on the excitation of carriers by laser pulse, which results in current surge and thus leads to the radiation of terahertz signals. ${ }^{5}$ Another example is terahertz radiation from nonlinear crystals by optical rectification. ${ }^{6}$ Other techniques have also been widely discovered and studied, such as terahertz emission through air plasma, ${ }^{7}$ radiation from superconducting thin films, ${ }^{8}$ and recently the terahertz generation from aligned molecules due to intramolecular microscopic charge transfer. $^{9}$

It has been known that some metal films (gold or silver) with particular nanostructured surfaces illuminated by femtosecond laser pulse can exhibit strong field enhancement due to the resonantly driven oscillation of collective electrons, which is known as surface plasmons (SPs).$^{10}$ The coupling from incident photons into SP waves causes a high confinement of electromagnetic energy to subwavelength spatial region, which creates field enhancement near the surfaces and thus introducing multiphoton photoelectric emission effect. Previous studies have indicated that surface with nanostructures could significantly contribute to the surface

\footnotetext{
${ }^{a)}$ Author to whom correspondence should be addressed. Electronic mail: sxy105@psu.edu.
}

field enhancement and thus improve the multiphoton photoelectric effect. ${ }^{11}$ Subsequently, we believe that this effect might also contribute to the enhancement of terahertz radiation. Although recent study and experiments have reported the observation of terahertz signal from metal grating due to SP excitation, ${ }^{12}$ no other types of nanostructured surfaces have been studied and reported for the enhanced terahertz generation associated with SP excitation under a femtosecond laser stimulation. Furthermore, the relationship between terahertz radiation and the surface nanostructures has not been systematically and thoroughly studied yet.

In this paper, a systematical study is conducted on the physical mechanism of terahertz generation in the nanostructured metal thin films, which can offer a valuable guide to maximize the terahertz generation. The physical mechanism behind this is strongly related to the multiphoton photoelectric effect. To further enhance terahertz signal strength and to better understand the mechanism of terahertz wave generation through the excitation of SP, we will quantitatively simulate and compare the strength of terahertz signal generated by different types of metal (gold) surfaces including flat film, grating, nanoparticles, nanoparticle rings, and pyramidshaped particles. We will also study how the near field enhancement and the subsequent multiphoton emission effect can be affected by each specific type of nanostructured surface. We will construct a theoretical physical model to describe the electron dynamics near metal film surfaces and the corresponding far field terahertz radiation. To validate this model, the results of the simulated terahertz radiation will be compared with the previously reported experimental results. $^{12,13}$ 


\section{PHYSICAL MODEL OF TERAHERTZ RADIATION DUE TO THE SP EXCITATION ON NANOSTRUCTURED SURFACES}

Photoemission process can happen either linearly or nonlinearly, which is decided by the choice of the wavelength of incident laser and the metal films' work function. The gold film, which is typically used to excite SP waves, has a work function of $5.3 \mathrm{eV},{ }^{14}$ while a typical Ti:sapphire laser used for terahertz generation through SP excitation has a wavelength around $800 \mathrm{~nm}$, which corresponds to a photon energy of $1.5 \mathrm{eV}$. Therefore, the eighth-order multiphoton photoelectric effect might be responsible for terahertz radiation. Surface roughness of metal film could effectively lower the work function, ${ }^{11}$ and thus the number of emitted oscillating electrons contributing to terahertz radiation will be larger than that on flat surfaces. We believe that the photoelectric emission effect, which determines the strength of terahertz radiation, is closely related to the strength of electric field inside the metal, and therefore it needs to be carefully investigated. We use the electric field inside a metal film as the relative measurement of the number of emitted electrons. Let the number of emitted electrons contributing to terahertz radiation be $N(t) \propto[E(t)]^{n}$, where $E(t)$ is the time dependent electric field inside the metal film associated with the SP excitation. For flat surface gold film, previous study has shown the eighth-order photoelectric emission process, which indicates that $n$ should be chosen as $8 .^{14}$ We assume that electrons emitted outside the metal surface are free electrons. This is because the collision frequency of electrons in vacuum is low, and the subsequent electron dynamics is governed by the electric and magnetic fields near the surfaces associated with the excitation of SP through Lorentz force equation. Moreover, in order to simplify our model, the interaction among electrons is ignored, this assumption is valid when the highest current density created by the laser source does not preponderate over the space charge saturation. ${ }^{15}$ The complexity existing in our model is the fact that analytical solutions regarding the electromagnetic fields inside the metal and near the surfaces cannot be acquired. Instead, we have to numerically solve Maxwell's equations and the Lorentz force equation to obtain the fields and the electron dynamics.

First, we need to calculate the electromagnetic fields on both sides of metal surfaces with each specific type of nanostructure by solving Maxwell's equations and boundary conditions. After acquiring the electric and magnetic fields in association with the excitation of SP, electrons are situated close to the metal film surfaces representing the electron motions when subjected to the fields. Those electron dynamics are dominated by the Lorentz force equation ${ }^{15}$

$$
\frac{\partial v}{\partial t}=\frac{e}{m}\left(E+\mu_{0} v \times H\right),
$$

where $E$ and $H$ are the electric and magnetic fields, respectively, $e / m$ is the ratio of charge to mass, and $v$ is the electrons' velocity. By solving this equation, the acceleration and velocity of the electrons could be obtained at each point spatially and temporally.
Then we use Hertzian dipole antenna approximation to account for the radiated far field; the corresponding electric field can be described as ${ }^{16}$

$$
E_{\mathrm{THz}}(t) \propto \frac{\partial J(t)}{\partial t},
$$

where $J(t)$ is the photocurrent density, which represents the convolution of optical intensity profile of the incident laser and of the impulse response of this SP excitation system and it can be written as ${ }^{17}$

$$
J(t)=I_{\text {laser }}(t) \otimes[e \cdot N(t) \cdot v(t)],
$$

where $\otimes$ indicates a convolution product; $I_{\text {laser }}(t)$ is the incident optical intensity profile; and $N(t), v(t)$, and $e$ are electron density, velocity, and electron charge, respectively. Here $N(t) \propto[E(t)]^{n}, n$ is chosen to be $8,{ }^{14}$ and $E(t)$ represents the electric field inside the metal film. Because of the fact that the electric field in this region plays an important role in pushing electrons out of the gold film and thus is related to the photoelectric emission effect. The product $e \cdot N(t) \cdot v(t)$ denotes the impulse response of the SP excitation system, such as the response to delta-function-like optical pumping. The incident optical intensity profile is assumed to be Gaussian in this model. The electron density $N(t)$ is not identical spatially and temporally during the excitation, depending on the electric field intensity inside the metal. The nonlinear term $N(t) \cdot v(t)$ introduces the possibility for frequency mixing and plays a major role in the terahertz emission.

As for different nanostructures used in this paper, they all need to satisfy the conditions for the excitation of SP. We use the Kretschmann geometry for a flat gold film structure. In order to couple the incident light into SP waves, the incident angle and the wavelength need to be chosen to satisfy the optimum coupling equation ${ }^{10}$

$$
\sqrt{\varepsilon_{d}} \frac{\omega}{c} \sin \theta=k_{\mathrm{SP}}
$$

and

$$
k_{\mathrm{SP}}=\frac{\omega}{c}\left(\frac{\varepsilon_{m} \varepsilon_{d}}{\varepsilon_{m}+\varepsilon_{d}}\right)^{1 / 2},
$$

where $k_{\mathrm{SP}}$ is the wave number of SP, and $\varepsilon_{d}$ and $\varepsilon_{m}$ are the permittivities of glass and gold, respectively. $\omega$ is the angular frequency of incident light and $\theta$ is the incident angle. For the other periodic structures, the following equations need to be satisfied for the coupling from the incident light to the SP waves: ${ }^{10}$

$$
\sqrt{\varepsilon_{d}} \frac{\omega}{c} \sin \theta=k_{\mathrm{SP}}+\Delta k_{p},
$$

where $\Delta k_{p}$ stems from any perturbation on the surfaces and is defined as $\Delta k_{p}=2 \pi / \Lambda$, and $\Lambda$ is the spatial period.

In order to closely resemble the actual experimental situation, femtosecond laser pulses with a $100 \mathrm{fs}$ pulse duration are used in our simulation, which impinges on the glass metal interface with a certain incident angle. The wavelength of the laser will be chosen to satisfy the resonance condition. 


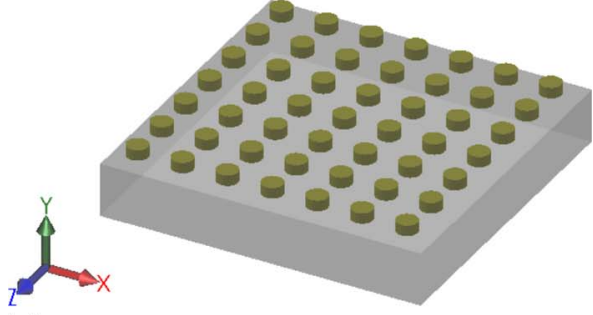

(a)

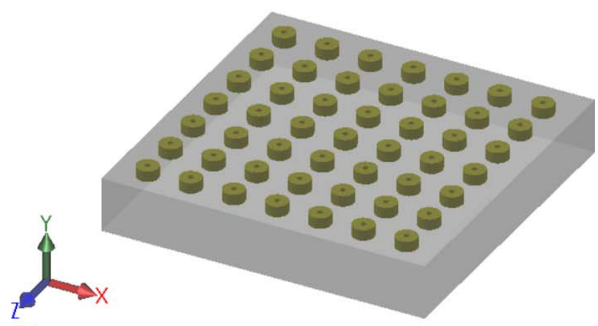

(b)

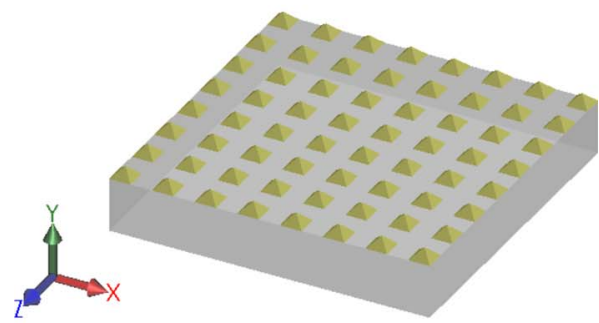

(c)

FIG. 1. (Color online) $[(a)-(c)]$ Geometry of the surface structures used to generate terahertz radiation.

In order to make a clear comparison between each surface structure and in an attempt to find the optimum one to generate the strongest terahertz radiation, we vary the key parameters for each geometry: (a) For a flat gold film, the film thicknesses used are $d=20,30,40$, and $50 \mathrm{~nm}$, and the incident angle is set to be $\theta=45^{\circ}$. (b) For metal gratings, a $30 \mathrm{~nm}$ layer of gold is deposited on the glass with grating constants of 400,700 , and $900 \mathrm{~nm}$. The ridge of the grating is $10 \mathrm{~nm}$ high, and it has a 50\% duty cycle. The incident angle is set to be $\theta=45^{\circ}$. (c) For nanoparticle structures, we investigate both single nanoparticle and nanoparticle arrays. All nanoparticles are disk shaped with $150 \mathrm{~nm}$ in diameter and $14 \mathrm{~nm}$ in thickness. The nanoparticle arrays are square lattices of disks with periodic distances of 500, 550, and $600 \mathrm{~nm}$, respectively. (d) For nanoparticle rings, in order to clearly compare the terahertz radiation by using this structure with that by using the structure of (c), the parameters chosen for this structure are the same as that in structure (c) with the only difference that each particle has a hollow core of $20 \mathrm{~nm}$ in diameter. (e) For pyramid-shaped particles, the height of the particle is $14 \mathrm{~nm}$ and the undersurface has a square area of $150 \times 150 \mathrm{~nm}^{2}$. The particle arrays are also square lattices with periodic distances of 500, 550, and $600 \mathrm{~nm}$, respectively. Normal incident angle is used for the structures of (c)-(e). The geometry and dimensions of these three nanostructures used to generate terahertz radiation are shown in Figs. 1(a)-1(c), respectively.
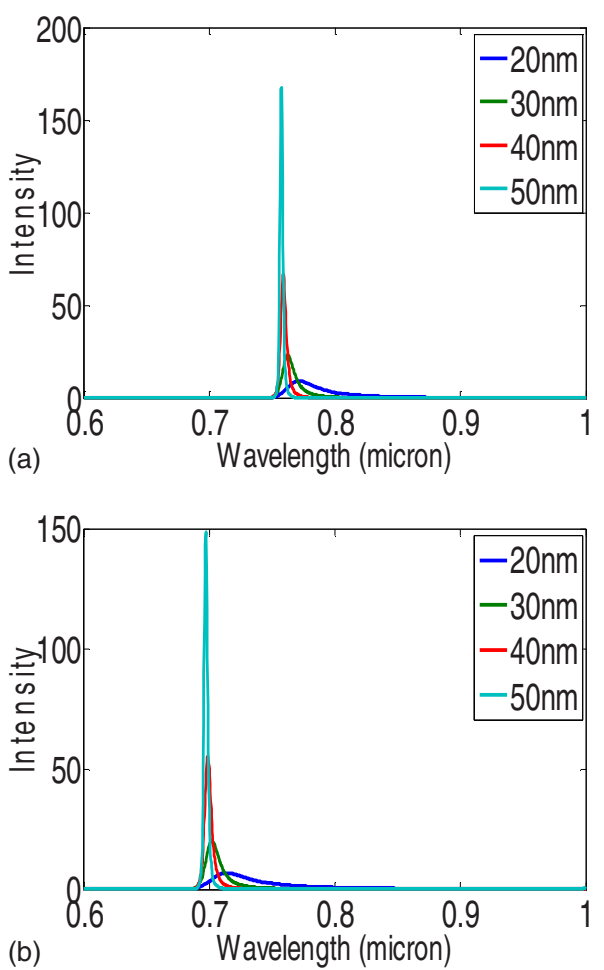

FIG. 2. (Color online) (a) Electric field intensity spectrum obtained inside a gold film (2.5 nm far from the surface inside the metal) for different film thicknesses $(d=20,30,40$, and $50 \mathrm{~nm}$, respectively). The incident angles are set $\theta=45^{\circ}$. (b) Electric field intensity spectrum obtained inside a gold film $(2.5 \mathrm{~nm}$ far from the surface inside the metal) for different film thicknesses $(d=20,30,40$, and $50 \mathrm{~nm}$, respectively). The incident angles are set $\theta$ $=50^{\circ}$.

\section{SIMULATION RESULTS AND DISCUSSIONS}

For the flat gold film surface structures, Fig. 2(a) illustrates the electric intensity inside the film as a function of wavelength for a fixed incident angle $\left(\theta=45^{\circ}\right)$ for four different thicknesses of the film. By varying the wavelength of the laser source (from 600 to $1000 \mathrm{~nm}$ and the amplitude is normalized to 1), we find that as the gold film becomes thicker, the resonant field intensity increases and the resonance wavelength has a weak blueshift. The resonant wavelengths are 771, 763, 759, and $755 \mathrm{~nm}$ for the film thicknesses of 20,30, 40, and $50 \mathrm{~nm}$, respectively. From these results, we believe that the incident angle and light wavelength should both be optimized for each film thickness in order to satisfy the optimum coupling equation so that the electric field inside the metal, which is related to the photoelectric emission effect, can be greatly enhanced and thus the strongest terahertz radiation could occur. Note that Fig. 2(a) was obtained with a fixed incidence angle $\left(\theta=45^{\circ}\right)$, and therefore only the optimum wavelength was obtained for each film thickness. In order to better explain the optimum matching condition between the incident angle and wavelength, Fig. 2(b) also shows the electric intensity spectrum for the incident angle $\theta=50^{\circ}$. Clearly, the resonant wavelength is blueshifted for the larger incident angle. This can be explained by the optimum coupling equation, which shows that for each incident angle, the matching condition is satisfied for a single resonant wavelength, and also, the resonant wavelength decreases with increasing the incident angle. 

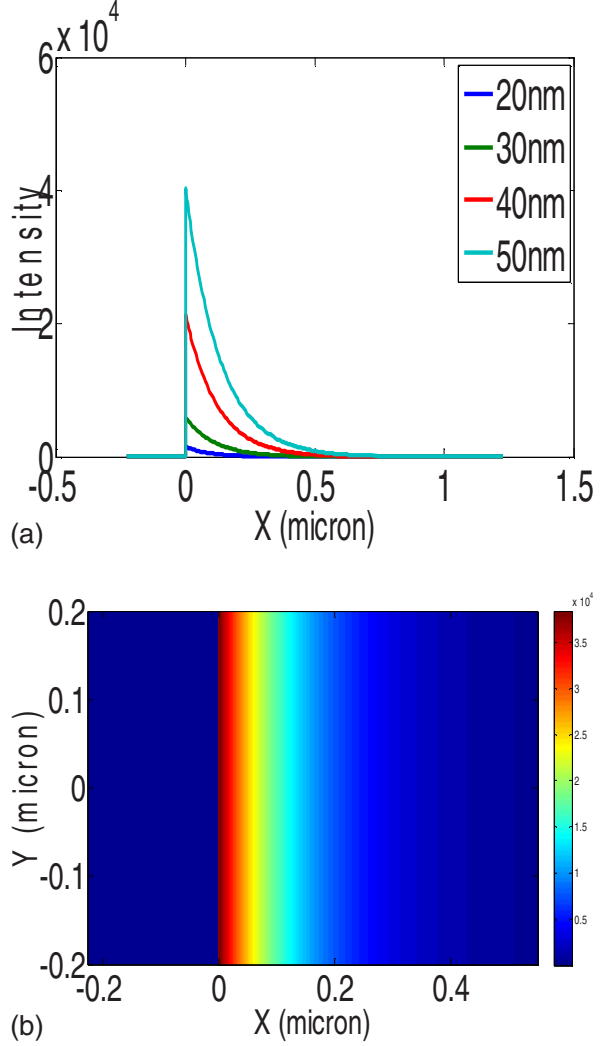

FIG. 3. (Color online) (a) Electric field intensity along the $x$ direction perpendicular to the surfaces for different film thicknesses when satisfying both the incident angle $\left(\theta=45^{\circ}\right)$ and resonant wavelength $(\lambda=755 \mathrm{~nm})$ condition. ( $x=0$ indicates the interface between metal and vacuum). (b) Electric field intensity near the metal vacuum interface (the film thickness is $50 \mathrm{~nm}$ ) when satisfying both the incident angle $\left(\theta=45^{\circ}\right)$ and resonant wavelength $(\lambda=755 \mathrm{~nm})$ condition $(x=0$ indicates the interface between metal and vacuum).

Figure 3(a) shows the electric field intensity as a function of the position in the direction normal to the surface due to the excitation of SP [Fig. 3(b) is another view of this effect]. This surface field distribution follows $E_{\mathrm{SP}}(x, t)$ $=\eta E_{\text {laser }}(t) \exp (-\alpha x),{ }^{14}$ where $\eta$ is the enhancement factor, $E_{\text {laser }}$ is the electric field of incident light, and $\alpha$ indicates exponentially decayed electric field into vacuum along the direction normal to the surfaces. The electric intensity on the left hand side of the metal vacuum interface $(x<0)$, which indicates the field inside a metal film, is negligibly small compared with the field in the vacuum region near the surfaces. However, this does not mean the electric intensity inside the metal film is zero. Due to the importance of the electric field inside the metal, which significantly affects the photoelectric emission effect and thus the strength of terahertz radiation, we need to focus our attention in this region and investigate the field intensity in detail. Based on the zoomed-in view shown in Fig. 4, we find that the electric intensity inside the metal film gradually increases with the position $x$ until $x=-1 \mathrm{~nm}$. An abrupt increase in the field intensity happens between $x=-1$ and $x=0$. We believe that the electrons situated in this narrow region $[x \in(-1,0)]$ provide the most important contribution to photoelectric emission effect due to the fact that they are subjected to a much stronger electric field that can easily push electrons out of the

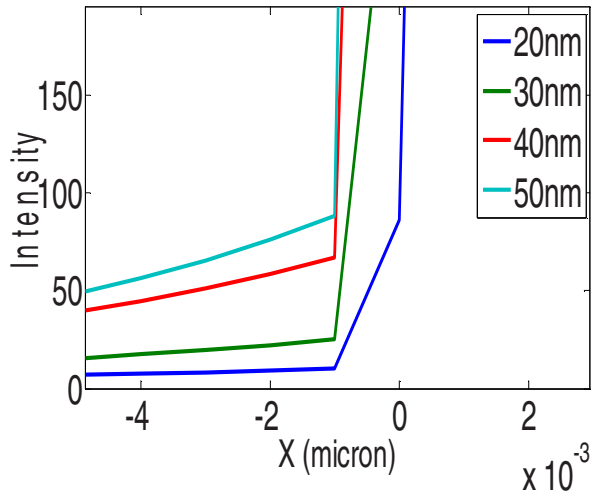

FIG. 4. (Color online) Electric field intensity inside the metal film along the $x$ direction perpendicular to the surfaces for different film thicknesses when satisfying both the incident angle $\left(\theta=45^{\circ}\right)$ and resonant wavelength $(\lambda$ $=755 \mathrm{~nm})$ condition $(d=20,30,40$, and $50 \mathrm{~nm}$, respectively, and $x<0$ indicates the region inside the metal).

metal film. However, if the incident angle does not match the optical signal wavelength to satisfy the optimum coupling condition, the efficiency of coupling from the incident photons to the SP waves can be very low, and the incident light will be mostly reflected back to the prism, which can be seen from Figs. 5(a) and 5(b). This observation verifies that the optimum coupling condition from the incident photons to the SP waves depends both on the incidence angle and the optical signal wavelength. A comparison between Figs. 6 and 4 makes it clear that the electric intensity inside the metal film may become much smaller if the optimum coupling condi-
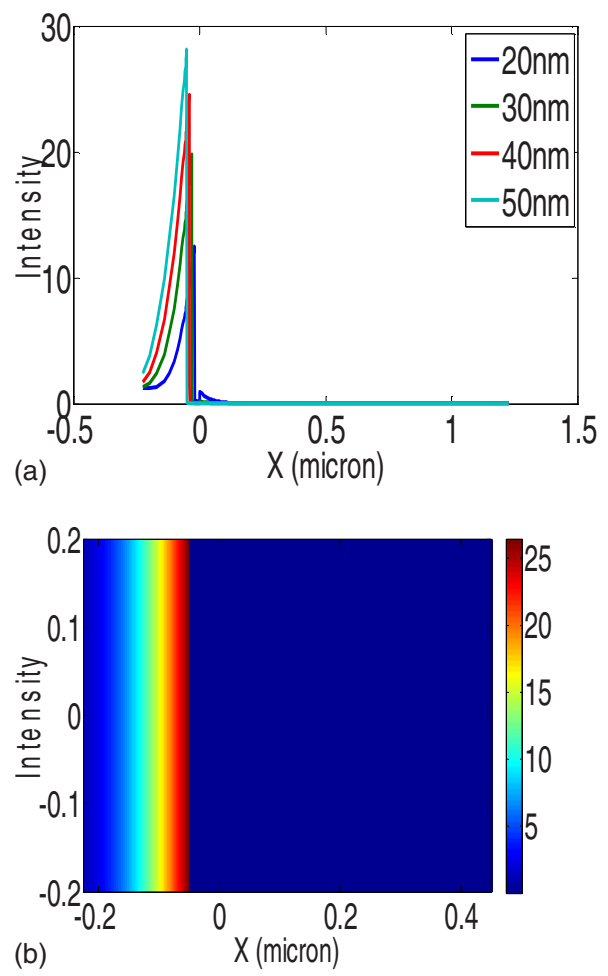

FIG. 5. (Color online) (a) Electric field intensity along the $x$ direction perpendicular to the surfaces for different film thicknesses $(d=20,30,40$, and $50 \mathrm{~nm}$, respectively), if the incident wavelength $(\lambda=1 \mu \mathrm{m})$ does not match with the incident angle $\left(\theta=45^{\circ}\right)$. (b) Electric field intensity near the metal vacuum interface (the film thickness $d=50 \mathrm{~nm}$ ), if the incident wavelength $(\lambda=1 \mu \mathrm{m})$ does not match with the incident angle $\left(\theta=45^{\circ}\right)$. 


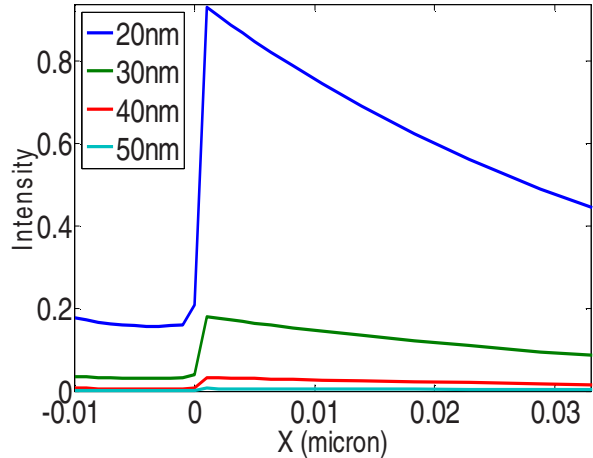

FIG. 6. (Color online) Electric field intensity inside the metal film along the $x$ direction perpendicular to the surfaces for different film thicknesses $(d$ $=20,30,40$, and $50 \mathrm{~nm}$, respectively $)$, if the incident wavelength $(\lambda$ $=1 \mu \mathrm{m})$ does not match with the incident angle $\left(\theta=45^{\circ}\right)$.

tion is not satisfied, which will significantly reduce the efficiency of photoelectric emission and thus producing much weaker terahertz radiation. Figure 7 shows the spectrum of far field terahertz radiation created by films with various thicknesses. The largest peak value is $\sim 11 \times 10^{4}$ by using a $40 \mathrm{~nm}$ thick gold film. However, according to Fig. 4, the largest electric field inside the metal film is created by using the film with a thickness of $50 \mathrm{~nm}$; this would imply that the strongest terahertz signal should be generated when the film thickness was $50 \mathrm{~nm}$. This inconsistency may be explained by the following: The radiated terahertz signal strength is affected not only by the electric field inside the metal film but also by the velocity of electrons, which is proportional to the ponderomotive force when electrons are pushed out of the surface and the fields near the surface. It is important to note that the peak values shown in Fig. 7 only provide relative strengths instead of absolute values. This is because we use two proportional relationships $N(t) \propto[E(t)]^{n}$ and $E_{\mathrm{THz}}(t)$ $\propto \partial J(t) / \partial t$ instead of exact equations. However, this is sufficient for the purpose of comparing terahertz generation efficiencies using different surface nanostructures.

As for grating nanostructures on the metal surface, we simulated terahertz radiation efficiencies using grating constants of 400, 700, and $900 \mathrm{~nm}$, respectively. Figure 8 illustrates the electric field intensity, which is indicated by the density of lines, near the surfaces associated with the excita-

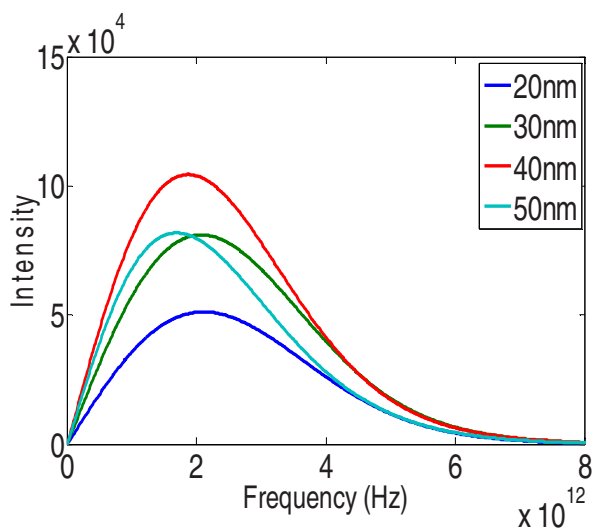

FIG. 7. (Color online) Terahertz radiation in the far field for different film thicknesses $(d=20,30,40$, and $50 \mathrm{~nm}$, respectively).

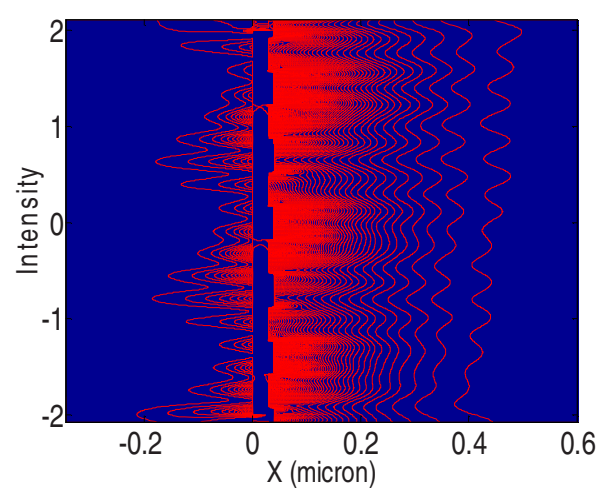

FIG. 8. (Color online) Electric field intensity near the metallic surface (the grating constant is $700 \mathrm{~nm})$, when the incident wavelength $(\lambda=752 \mathrm{~nm})$ matches with the incident angle $\left(\theta=45^{\circ}\right)$.

tion of SP. Other parameters of the grating are as follows: The duty cycle is $50 \%$, the depth is $10 \mathrm{~nm}$, and the thicknesses of shallow and ridge portions are 30 and $40 \mathrm{~nm}$, respectively. Field enhancement effect near the surface is obvious in this figure, and the field intensity near the corner of the ridges in each grating period is the strongest due to the point discharge effect. Therefore it is fair to assume that photoelectric emission can happen more easily for electrons located in this region, and consequently these electrons have more important contributions to the terahertz generation. Figure 9 shows the emitted terahertz spectral densities using grating structure with different grating constants. This simulated terahertz emission is consistent with the experimental result of Fig. 3 in Ref. 12. Also, Comparing Fig. 9 with Fig. 7 , it is evident that the grating structure on the surface enhances terahertz generation by approximately three times. Note that, in the calculation, the film thickness under the grating is $30 \mathrm{~nm}$. Also, for Fig. 9, the emitted terahertz signal is measured in the far field by using Eq. (2), and for Fig. 8, the electric field is calculated near the grating surface. This enhanced efficiency is attributed to the stronger internal electric field, which causes more electrons to be pushed out of the surface and thus contributing to terahertz radiation. This might explain why it has been difficult to observe terahertz signal experimentally by using flat film surfaces.

To investigate the possibility of further terahertz emis-

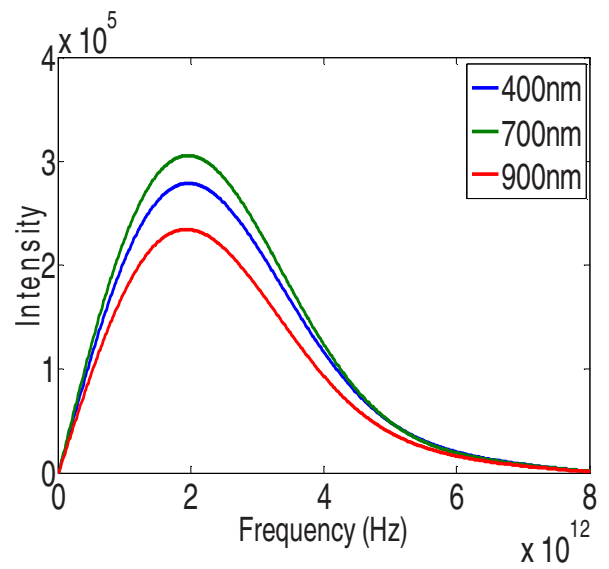

FIG. 9. (Color online) Terahertz radiation by using grating structures for different grating constants ( $d=400,700$, and $900 \mathrm{~nm}$, respectively) 


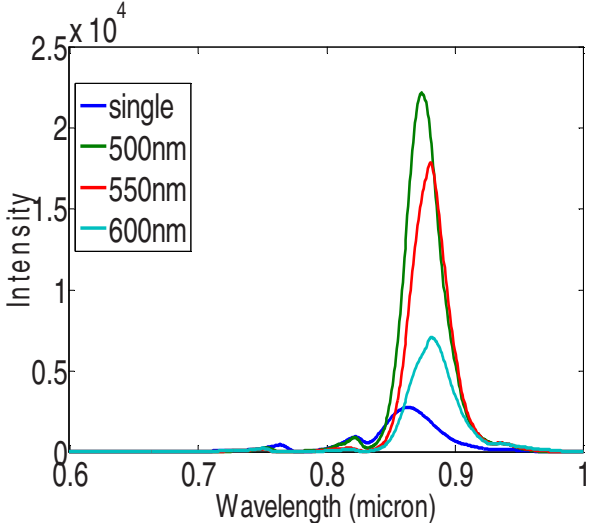

FIG. 10. (Color online) Electric field intensity spectrum obtained inside the metal ( $1 \mathrm{~nm}$ far from the surface inside the metal) for different nanoparticle structures (single nanoparticle, $d=500,550$, and $600 \mathrm{~nm}$ ). The incident angles are set to be normal.

sion enhancement by employing nanostructured surface, several types of nanostructured surfaces are selected for simulation. The following parameters are used in simulating the nanoparticle structured surface: Nanoparticles have a disk shape with $150 \mathrm{~nm}$ in diameter and $14 \mathrm{~nm}$ in thickness. The nanoparticle arrays are square lattices of disks. Also, different periods, 500, 550, and $600 \mathrm{~nm}$, are employed during the simulation. Normal incident angle is used for the excitation. Figure 10 shows the calculated resonance wavelength spectrum for several different surface lattice structures. In comparison with Fig. 2, the dramatic difference in this structure is that the field intensity at the resonance wavelength becomes much more pronounced than the flat films. It also indicates that lattice structured nanoparticle array on the metal surface may significantly enhance the terahertz field intensity in comparison to that with a single nanoparticle. Figure 11 illustrates the variation of the electric field intensity along the direction normal to the metal surfaces, which shows a secondary peak corresponding to the surface position of nanoparticles. Although the field enhancement near nanoparticle surfaces is similar to that in a flat gold film (the field intensities for both cases are on the order of $10^{4}$ ), the pronounced distinction between the flat surface and the one with nanoparticles is that the electric field intensity inside the

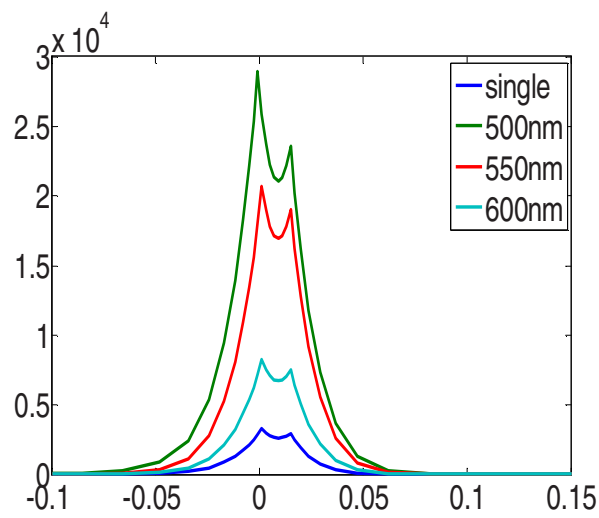

FIG. 11. (Color online) Electric field intensity along the $z$ direction perpendicular to the surfaces for different nanoparticle structures $(0<z$ $<0.014 \mu \mathrm{m}$ indicates the region inside the metal).

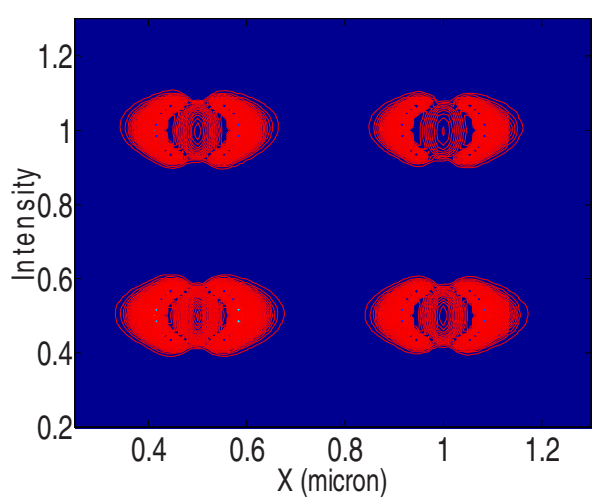

FIG. 12. (Color online) Electric field intensity near the surfaces of gold disks (15 $\mathrm{nm}$ far from the nanoparticle surface), when satisfying the resonant incident wavelength $(\lambda=880 \mathrm{~nm})$. The distance between those disks $d=500 \mathrm{~nm}$.

metal is roughly two orders of magnitude higher when nanoparticles are introduced on the surface. It is the dramatically increased electric field intensity inside the metal that pushes much more electrons out of the metal surface and thus producing much stronger terahertz radiation. We believe that this enhanced field intensity inside the metal film with nanostructured surfaces is due to the strong constructive interference of the electric field between particles. Figure 12 shows the calculated electric field intensity distribution near the surfaces outside the metal film (measured $15 \mathrm{~nm}$ from the surface), which is indicated by the density of lines. The field distribution appears highly localized with the decay distance in the order of several tens of nanometers. This high confinement of energy could be another reason to explain the strong electric field inside the metal. Figure 13 shows the far field terahertz radiation spectrum from surfaces with nanoparticle structures. This simulated terahertz radiation is in good agreement with the experimental result of Fig. 3(b) in Ref. 13. The largest peak value in this figure is approximately $\sim 15 \times 10^{6}$, which is more than two orders of magnitude higher than that produced from a flat film, and about 40 times higher than the one from a surface with a grating structure.

Furthermore, in order to get stronger terahertz radiation, some other surface structures, such as nanoparticle rings and

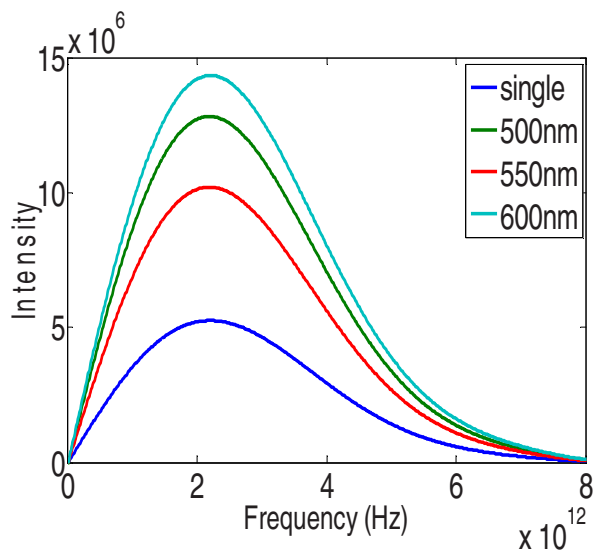

FIG. 13. (Color online) Terahertz radiation in the far field for nanoparticle structures (single nanoparticle, $d=500,550$, and $600 \mathrm{~nm}$, respectively). 


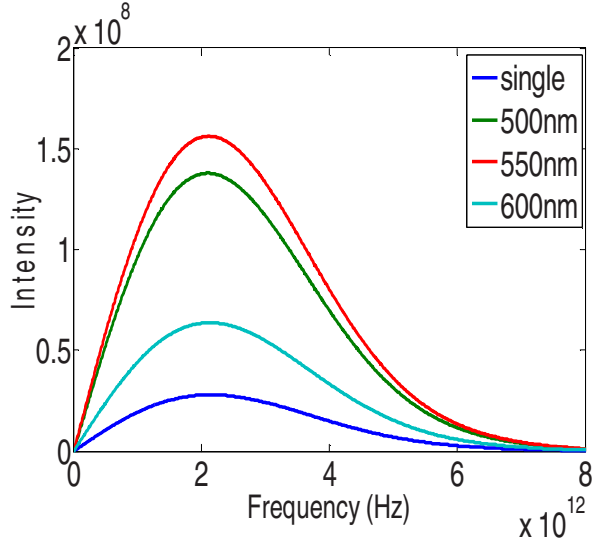

FIG. 14. (Color online) Terahertz radiation in the far field for nanoparticle ring structures (single nanoparticle ring, $d=500,550$, and $600 \mathrm{~nm}$, respectively).

pyramid-shaped particles, are also investigated here. Figures 14 and 15 depict the calculated terahertz emission spectrum by using these two kinds of nanostructures. The results indicate that terahertz radiation strength could be more enhanced by using nanoparticle rings to a peak value of $\sim 16 \times 10^{7}$, which is even one order higher than the nanoparticle arrays, while this strength drops greatly by using pyramid-shaped particles. This dramatic difference indicates that terahertz generation efficiency could be affected by the shape of the particles composing the surface structure. The anticipated underlying reason is the great difference of electric field magnitude inside the metal for different types of nanostructures, which governs the photoelectric emission effect and thus the strength of terahertz radiation. In the future, we will make a thorough investigation on the enhancement mechanism for each type of nanostructure and try more surface nanostructures and identify the optimum nanostructure so that terahertz generation efficiency can be further improved.

Through the analysis of terahertz generation on metal films with five different surface structures, the mechanism becomes clear: Although the electron velocity outside the metal surface may affect the terahertz generation efficiency

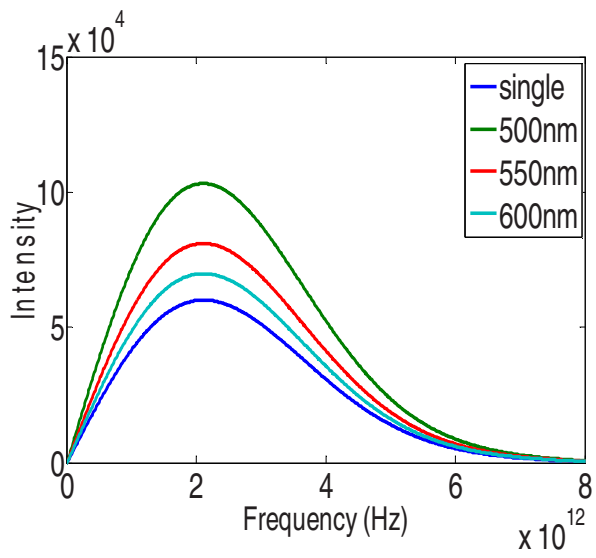

FIG. 15. (Color online) Terahertz radiation in the far field for pyramidshaped particle structures (single particle, $d=500,550$, and $600 \mathrm{~nm}$ ). to some extent as indicated by Fig. 7, this efficiency is predominantly determined by the electric field magnitude inside the metal film. The evidence of the enhanced terahertz signal intensity on metal film surfaces with nanostructures provided by our analysis may open the possibility of creating higher efficiency terahertz sources and sensors with nanoengineered metal surfaces.

\section{CONCLUSION}

We have developed a SP based photoemission model for systematic investigation of terahertz radiation from nanostructured metal films. The electric field intensities both inside and outside the metal were investigated in the vicinity of the surface since the fields in both of these two regions played important roles in the terahertz generation. Theoretically speaking, the number of emitted electrons contributing to terahertz generation was determined by electric field inside the metal, whereas the quivering motions of electrons after they were pushed out of metal were governed by the electromagnetic field immediately outside the surface. Thus, an artificial nanostructure on the metallic surface may play an important role for photoelectric emission and thus affect the strength of the radiated terahertz signal. The study presented in this paper not only provides an effective tool for analyzing the SP based terahertz generation, but also offers a useful guidance on how to generate stronger terahertz signal by optimizing the nanostructures of the metal surfaces.

\section{ACKNOWLEDGMENTS}

The authors greatly acknowledge the partial financial support of this work by ONR basic research program.

${ }^{1}$ G. H. Welsh and K. Wynne, Opt. Express 17, 2470 (2009).

${ }^{2}$ W. L. Chan, M. L. Moravec, R. G. Baraniuk, and D. M. Mittleman, Opt. Lett. 33, 974 (2008).

${ }^{3}$ G. M. Png, R. Flook, B. W. H. Ng, and D. Abbott, Electron. Lett. 45, 343 (2009).

${ }^{4}$ M. Naflaly and R. E. Miles, Proc. IEEE 95, 1658 (2007).

${ }^{5}$ M. Tani, M. Herrmann, and K. Sakai, Meas. Sci. Technol. 13, 1739 (2002).

${ }^{6}$ D. H. Auston, K. P. Cheung, J. A. Valdmanis, and D. A. Kleinman, Phys. Rev. Lett. 53, 1555 (1984).

${ }^{7}$ H. Zhong, N. Karpowicz, and X. C. Zhang, Appl. Phys. Lett. 88, 261103 (2006).

${ }^{8}$ M. Hangyo, S. Tomozawa, and Y. Murakami, Appl. Phys. Lett. 69, 2122 (1996).

${ }^{9}$ J. J. Carey, R. T. Bailey, D. Pugh, J. N. Sherwood, F. R. Cruickshank, and K. Wynne, Appl. Phys. Lett. 81, 4335 (2002).

${ }^{10}$ H. Raether, Surface Plasmons on Smooth and Rough Surfaces and on Gratings (Springer-Verlag, Berlin, 1988).

${ }^{11}$ M. Aeschlimann, C. A. Schmuttenmaer, H. E. Elsayed-Ali, and R. J. D. Miller, J. Chem. Phys. 102, 8606 (1995).

${ }^{12}$ G. H. Welsh, N. T. Hunt, and K. Wynne, Phys. Rev. Lett. 98, 026803 (2007).

${ }^{13}$ X. J. Wu, X. S. Chen, Y. Zhao, S. He, F. L. Zhao, G. Wang, IRMMW-THz, pp. 464-465 (2007).

${ }^{14}$ H. L. Skriver and N. M. Rosengaard, Phys. Rev. B 46, 7157 (1992).

${ }^{15}$ S. E. Irvine and A. Y. Elezzabi, Phys. Rev. A 73, 013815 (2006).

${ }^{16}$ K. Sakai, Terahertz Optoelectronics (Springer-Verlag, Berlin, 2005).

${ }^{17}$ L. Duvillaret, F. Garet, J. F. Roux, and J. L. Coutaz, IEEE J. Sel. Top. Quantum Electron. 7, 615 (2001). 